\title{
ON THE CORRECTNESS OF A NONLOCAL PROBLEM FOR THE SECOND ORDER MIXED TYPE EQUATION OF THE SECOND KIND IN A RECTANGLE
}

\author{
SIROZHIDDIN ZUHRIDDINOVICH DJAMOLOV* \\ The Institute of Mathematics at the National University of Uzbekistan, \\ 100125, Tashkent, Academgorodok, Durmon yuli street, 29. \\ "corresponding author: siroj@mail.ru \\ (Received: 15 th Apr. 2016; Accepted: $1^{\text {st }}$ Jul. 2016; Published on-line: $30^{\text {th }}$ Nov. 2016)
}

\begin{abstract}
In this work, the correctness of a nonlocal problem from the Sobolev spaces is proven under some restrictions to the coefficients of the considered second order mixed type equation of the second kind. The proof is accomplished with multiple methods namely, "$\varepsilon$-regularization", priory estimates, and the Galerkin method.
\end{abstract}

ABSTRAK: Dalam karya ini, ketepatan satu masalah bukan setempat dari ruang Sobolev telah dibuktikan di dalam beberapa sekatan kepada koefisien persamaan jenis bercampur perintah kedua daripada jenis yang kedua yang dipertimbangkan. Buktinya dicapai dengan pelbagai kaedah iaitu, "$\varepsilon$-regularization", anggaran priori dan kaedah Galerkin.

KEYWORDS: second order mixed type equation of the second kind; Sobolev space; Galerkin and $\varepsilon$-regularization methods

\section{INTRODUCTION}

The basic concepts of the theory of differential equations in partial derivatives were formed in the study of classical problems of mathematical physics and are currently well understood. However, the current problems of natural sciences lead to the necessity of setting and qualitatively studying new tasks, which is a striking example of a class of nonlocal problems.

As nonlocal problems we call such problems that determine relationships between the value of the solution or its derivatives at the boundary and interior points of a considered domain. In recent decades, nonlocal problems for partial differential equations are being actively studied by many mathematicians.

Among the first studies of non-local problems, we note a work by Bitsadze and Samarskii [4]. In this work, spatial and non-local problems for a certain class of elliptic equations were formulated and studied, which led to the study of non-self-adjoint spectral problems. Subsequently, the problem formulated in [4], has been named a BitsadzeSamarskii problem.

The investigation of nonlocal problems is caused by both theoretical interest and practical necessity. This is due to the fact that the mathematical models of various physical, chemical, biological, and ecological processes are often problems in which, instead of the classical boundary conditions, definite connection values of the unknown function (or its derivatives) on and within the boundary are given. Problems of this type may arise in the study of phenomena related to plasma physics, the spread of heat, the 
process of moisture transfer in capillary porous media issues, demographics, mathematical biology, and some technological processes. Nonlocal problems also have practical value in solving the problems of solid mechanics. They allow to control the stress-strain state and these are similar to the control tasks $[4,8-12,16,17,19]$.

For the first time, non-local problems in certain weight and negative spaces for equations of mixed type were investigated by functional methods in the works $[1,6,8,10$, $11,17]$.

\section{FORMULATION OF A PROBLEM}

In a rectangle $Q=(0,1) \times(0, T)$ we consider a second order differential equation

$$
L u=K(x, t) u_{t t}-u_{x x}+\alpha(x, t) u_{t}+c(x, t) u=f(x, t) .
$$

Problem: To find a generalized solution of Eq. (1) from the Sobolev space $W_{2}^{l}(Q),(2 \leq l$ is integer), satisfying boundary conditions

here $\gamma=$ const $\neq 0$.

$$
\begin{aligned}
& \gamma u(x, 0)=u(x, T), \\
& u(0, t)=u(1, t)=0,
\end{aligned}
$$

In the previous works $[6,7,11,17]$ the correctness of problem Eqs. (1)-(3) in the case where $K(x, 0)=K(x, T)=0$ was proven under comparatively strong conditions to the coefficients of Eq. (1). In the present work, we consider the case where, $K(x, 0) \leq 0 \leq K(x, T)$.

Note that Eq. (1) is the second kind of mixed type equation, since there is no restriction inside the domain $Q$ to the sign of the variable $t$ of the function $K(x, t)$ [5].

First, we consider the case where $l=2$. Assuming the coefficients of Eq. (1) are smooth enough and $\alpha(x, t), c(x, t)$ are periodic in function with respect to variable $t$.

\section{UNIQUENESS FOR PROBLEM}

\section{Theorem 1:}

Let aforementioned conditions to the coefficients of Eq. (1) are fulfilled, moreover let $2 \alpha-K_{t}+\lambda K \geq \delta_{1}>0 ; \lambda c-c_{t} \geq \delta_{2}>0$, where $\lambda=\frac{2}{T} \ln \gamma$, such that $\gamma \in(1, \infty)$. Then for any function $f(x, t) \in L_{2}(Q)$ a generalized solution of problem Eqs. (1)-(3) from the space $W_{2}^{2}(Q)$ is unique and the following inequality holds true

$$
\|u\|_{1} \leq m\|f\|_{0} .
$$

Here $(,)_{l}$ and \|\|$_{l}$ are respectively regular scalar product and norm of the Sobolev space $W_{2}^{l}(Q), 2 \leq l$ is an integer number and it is known that at $l=0, W_{2}^{0}(Q)=L_{2}(Q) \quad[2,5$, 13-15]. Here, and further, by $m$ we designate positive constants, the exact values of which are not of interest. 


\section{Proof:}

For any function $u \in W_{2}^{2}(Q)$, one can easily get the following equality by using integration by parts:

$$
\begin{aligned}
& 2 \int_{Q} L u \cdot \exp (-\lambda t) \cdot u_{t} d x d t=\int_{Q} \exp (-\lambda t)\left(2 \alpha-K_{t}+\lambda K\right) \cdot u_{t}^{2}+\lambda u_{x}^{2}+\left(\lambda c-c_{t}\right) u^{2} d x d t+ \\
& +\int_{\partial Q} \exp (-\lambda t)\left\{K u_{t}^{2} v_{0}-2 u_{x} u_{t} v_{1}+u_{x}^{2} v_{0}+c u^{2} v_{0}\right\} d s
\end{aligned}
$$

where $v=\left(v_{0}=\cos (v, t) ; v_{1}=\cos (v, x)\right)$ is a unit vector of the inner normal to the $\partial Q$. Conditions of Theorem 1 guarantee non-negativity of the integral over the domain $Q$. Let $u \in W_{2}^{2}(Q)$ satisfy boundary conditions Eqs. (2)-(3), on condition that Theorem 1, with respect to function $K(x, t)$, must meet the condition of $K(x, 0) \leq 0 \leq K(x, T)$. With respect to function $c(x, t)$, the periodicity of variables $t$ and $\gamma^{2}=e^{\lambda T}$ satisfy conditions Eqs. (2)-(3), thereby leading to positivity of the following boundary integral.

$$
\begin{aligned}
& J=\int_{\partial Q} \exp (-\lambda t)\left\{K u_{t}^{2} v_{0}-2 \cdot u_{x} u_{t} v_{1}+u_{x}^{2} v_{0}+c \cdot u^{2} v_{0}\right\} d s=\int_{\partial Q} \exp (-\lambda t)\left\{K u_{t}^{2}+u_{x}^{2}+c u^{2}\right\} v_{0} d s- \\
& -2 \int_{\partial Q} u_{x} u_{t} v_{1} d s=\int_{0}^{1}\left[K(x, T) e^{-\lambda T} \gamma^{2}-K(x, 0)\right] u_{t}^{2}(x, 0) d x+\left[e^{-\lambda T} \gamma^{2}-1\right] \int_{0}^{1} u_{x}^{2}(x, 0) d x+ \\
& +\int_{0}^{1}\left[c(x, T) e^{-\lambda T} \gamma^{2}-c(x, 0)\right] u^{2}(x, 0) d x-2 \int_{0}^{T}\left[u_{x}(0, t) u_{t}(0, t)-u_{x}(1, t) u_{t}(1, t)\right] d t \ldots \\
& . . \int_{0}^{1}[K(x, T)-K(x, 0)] u_{t}^{2}(x, 0) d x+\int_{0}^{1}[c(x, T)-c(x, 0)] u^{2}(x, 0) d x . .0 .
\end{aligned}
$$

Omitting non-positive items from Eq. (4) we get

$$
2 \int_{Q} L u \cdot \exp (-\lambda t) u_{t} d x d t \geq \int_{Q} \exp (-\lambda t) \cdot\left\{\left(2 \alpha-K_{t}+\lambda K\right) u_{t}^{2}+\lambda u_{x}^{2}+\left(\lambda c-c_{t}\right) u^{2}\right\} d x d t
$$

Applying Young's inequality to Eq. (5) $[5,13,14]$ the following estimate is obtained

$$
\|u\|_{1}, m\|f\|_{0},
$$

from which the uniqueness of the solution of problem Eqs. (1)-(3) follows.

\section{EXISTENCE FOR PROBLEM}

In order to prove the existence of the solution of problem in Eqs. (1)-(3) in $W_{2}^{2}(Q)$ the "$" \varepsilon$-regularization" method is used with the Galerkin method [7, 13-15]. Consider a nonlocal problem for a composite type equation

$$
\begin{gathered}
L_{\varepsilon} u_{\varepsilon}=-\varepsilon \frac{\partial}{\partial t} \Delta u_{\varepsilon}+L u_{\varepsilon}=f(x, t) \\
\left.\gamma D_{t}^{q} u_{\varepsilon}\right|_{t=0}=\left.D_{t}^{q} u_{\varepsilon}\right|_{t=T} ; q=0,1,2 \\
u_{\varepsilon}(0, t)=u_{\varepsilon}(1, t)=0
\end{gathered}
$$


Here $D_{t}^{p} u=\frac{\partial^{p} u}{\partial t^{p}}, \quad D_{t}^{0} u=u, p=0,1,2, \Delta u=\frac{\partial^{2} u}{\partial t^{2}}+\frac{\partial^{2} u}{\partial^{2} x}$ is the Laplace operator in a plane, $\varepsilon$ is a small positive number, and $\gamma=$ const $\neq 0$ such that $\gamma \in(1, \infty)$.

Eq. (6) acts as an $\varepsilon$-regularization of Eq. (1) [5, 7, 13, 14]. The class of functions $u_{\varepsilon}(x, t) \in W_{2}^{2}(Q), \frac{\partial}{\partial t} \Delta u_{\varepsilon} \in L_{2}(Q)$ are designated through $V$, satisfying conditions in Eqs. (7), (8).

\section{Definition:}

As the regular solution of problem in Eqs. (6)-(8), function $u_{\varepsilon}(x, t) \in V$ satisfies Eq. (6).

\section{Theorem 2:}

Let the following conditions holds true in $\bar{Q}$ :

$$
2 \alpha-\left|K_{t}\right|+\lambda|K| \geq \delta_{1}>0 ; \lambda c-c_{t} \geq \delta_{2}>0,
$$

where $\quad \lambda=\frac{2}{T} \ln \gamma \quad$ such that $\gamma \in(1, \infty)$. Then for any function $f, f_{t} \in L_{2}(Q)$, $\gamma f(x, 0)=f(x, T)$ there exists a unique regular solution of problem in Eqs. (6)-(8) and the following inequalities are true:

$$
\begin{aligned}
& \text { I) } \varepsilon \cdot\left(\left\|\frac{\partial^{2}}{\partial t^{2}} u_{\varepsilon}\right\|_{0}^{2}+\left\|\frac{\partial^{2}}{\partial t \partial x} u_{\varepsilon}\right\|_{0}^{2}\right)+\left\|u_{\varepsilon}\right\|_{1}^{2}, m\|f\|_{0}^{2} ; \\
& \text { II) } \varepsilon \cdot\left\|\frac{\partial}{\partial t} \Delta u_{\varepsilon}\right\|_{0}^{2}+\left\|u_{\varepsilon}\right\|_{2}^{2}, m \cdot\left[\|f\|_{0}^{2}+\left\|f_{t}\right\|_{0}^{2}\right] .
\end{aligned}
$$

\section{Proof:}

The proof of inequality I) will be done similarly to the proof of Theorem 1, from which the uniqueness of the regular solution of problem in Eqs. (6)-(8) follows. Now, the second prior estimate is proven.

Let $\phi_{j}(x, t)$ are eigenfunctions of the following problem:

$$
\begin{gathered}
-\Delta \phi_{j}=\frac{\partial^{2} \phi_{j}}{\partial^{2} t}+\frac{\partial^{2} \phi_{j}}{\partial^{2} x}=\mu_{j}^{2} \phi_{j}, \\
\left.D_{t}^{p} \phi_{j}\right|_{t=0}=\left.D_{t}^{p} \phi_{j}\right|_{t=T} ; p=0,1, \\
\phi_{j}(0, t)=\phi_{j}(1, t)=0
\end{gathered}
$$

Due to the general theory of linear self-adjoint elliptic operators [2, 13, 15], it is known that all eigenfunctions of problem in Eqs. (9)-(11) form a fundamental system in $C^{\infty}(Q)$, which is orthogonal in $L_{2}(Q)$. Solving problems in Eqs. (9)-(11), is $\phi_{j}(x, t)=\cos \eta_{j} t \cdot \sin \tau_{j} x$, where $\eta_{j}=\frac{2 \pi j}{T} ; \tau_{j}=j \pi ; \mu_{j}^{2}=\eta_{j}^{2}+\tau_{j}^{2} ; j=0,1,2, \ldots$

Using these sequences of functions, a solution of the auxiliary problem is constructed 


$$
\begin{aligned}
& \ell \omega_{j}=e^{\frac{-\lambda t}{2}} \frac{\partial \omega_{j}}{\partial t}=\phi_{j}, \\
& \gamma \omega_{j}(x, 0)=\omega_{j}(x, T) .
\end{aligned}
$$

Obviously, problem in Eqs. (12)-(13) is uniquely solvable and its solution has the form

$$
\begin{aligned}
& \ell^{-1} \phi_{j}=\omega_{j}=\int_{0}^{t} \exp \left(\frac{\lambda \tau}{2}\right) \phi_{j} d \tau+\frac{1}{\gamma-1} \int_{0}^{T} \exp \left(\frac{\lambda t}{2}\right) \phi_{j} d t=\sin \tau_{j} x \cdot \int_{0}^{t} e^{\frac{\lambda \tau}{2}} \cos \eta_{j} t d t+\frac{1}{\gamma-1} \int_{0}^{T} e^{\frac{\lambda t}{2}} \cos \eta_{j} t d t= \\
& =\frac{e^{\frac{\lambda t}{2}}}{\eta_{j}^{2}+\frac{\lambda^{2}}{4}} \sin \tau_{j} x \cdot\left[\eta_{j} \sin \eta_{j} t+\cos \eta_{j} t-1\right]
\end{aligned}
$$

It is clear that the functions $\omega_{j}(x, t) \in C^{\infty}(Q)$ are linearly independent. Really, if $\sum_{j=1}^{m} c_{j} \omega_{j}=0$ for some set of sequences of functions $\omega_{1}, \omega_{2}, \ldots, \omega_{m}$, then acting on this sum by the operator $\ell$ results in

$$
\sum_{j=1}^{m} c_{j} \ell \omega_{j}=\sum_{j=1}^{m} c_{j} \phi_{j}=0
$$

From which follows that $c_{j}=0$ for any $j=\overline{1, m}$. Note, from the construction of function $\phi_{j}(x, t)$ the following conditions to the functions $\omega_{j}(x, t) \in C^{\infty}(Q)$ follow:

$$
\begin{gathered}
\left.\gamma D_{t}^{q} \omega_{j}\right|_{t=0}=\left.D_{t}^{q} \omega_{j}\right|_{t=q}, q=0,1,2 \\
\omega_{j}(0, t)=\omega_{j}(1, t)=0
\end{gathered}
$$

Now an approximate solution of Eqs. (6)-(8) is searched for in the form $w=u_{\varepsilon}^{N}=\sum_{j=1}^{N} c_{j} \omega_{j}$, where coefficients $c_{j} ; \forall j=\overline{1, m}$ are defined as solutions of the algebraic system.

$$
\int_{Q} L_{\varepsilon} u_{\varepsilon}^{N} \exp \left(-\frac{\lambda t}{2}\right) \phi_{j} d x d t=\int_{Q} f \exp \left(-\frac{\lambda t}{2}\right) \phi_{j} d x d t .
$$

The unique solvability of the algebraic system in Eq. (16) is proven. Multiplying every equation of (16) by $2 c_{j}$ and summarizing with respect to $j$ from 1 to $N$, considering problem in Eqs. (9)-(13) results in

$$
\int_{Q} L_{\varepsilon} w \exp (-\lambda t) w_{t} d x d t=\int_{Q} f \exp (-\lambda t) w_{t} d x d t .
$$

From which, by virtue of Theorem 2, by integrating the identity in Eq. (17) an approximate solution of problem in Eqs. (6)-(8) is obtained that estimates I) i.e.

$$
\varepsilon \cdot\left(\left\|\frac{\partial^{2}}{\partial t^{2}} u_{\varepsilon}\right\|_{0}^{2}+\left\|\frac{\partial^{2}}{\partial t \partial x} u_{\varepsilon}\right\|_{0}^{2}\right)+\left\|u_{\varepsilon}\right\|_{1}^{2} \leq m\|f\|_{0}^{2} .
$$

This implies the solvability of Eq. (16) $[6,8,13,14]$. In particular, from the estimate I) we obtain a weak solution of problem in Eqs. (6)-(8). 
Now the second priory estimate II) must be proven. Thanks to Eqs. (9)-(13), from the identity in Eq. (16) comes

$$
-\frac{1}{\mu_{j}^{2}} \int_{Q} L_{\varepsilon} w \exp \left(-\frac{\lambda t}{2}\right) \Delta \ell \omega_{j} d x d t=-\frac{1}{\mu_{j}^{2}} \int_{Q} f \exp \left(-\frac{\lambda t}{2}\right) \Delta \ell \omega_{j} d x d t .
$$

Multiplying each equation of (18) by $2 \mu_{j}^{2} c_{j}$ and summing $j$ from 1 to $N$, taking Eqs. (13),(14) into account from Eq. (18), the following is derived

$$
-2 \int_{Q} L_{\varepsilon} w e^{-\lambda t}\left[\frac{\partial \Delta w}{\partial t}-\lambda w_{t t}+\frac{\lambda^{2}}{4} w_{t}\right] d x d t=-2 \int_{Q} f e^{-\lambda t}\left[\frac{\partial \Delta w}{\partial t}-\lambda w_{t t}+\frac{\lambda^{2}}{4} w_{t}\right] d x d t
$$

Integrating Eq. (19) according to the conditions of Theorem 2 and the boundary conditions in Eqs. (14)-(15), we obtain the following inequality

$$
\begin{aligned}
& m\left[\left\|f_{t}\right\|_{0}^{2}+\|f\|_{0}^{2}\right] \geq \varepsilon\left\|\frac{\partial \Delta w}{\partial t}\right\|_{0}^{2}+\int_{Q} e^{-\lambda t}\left\{\left(2 \alpha+K_{t}-\lambda K\right) w_{t t}^{2}+\left(2 \alpha-K_{t}+\lambda K\right) w_{t x}+\lambda w_{x x}^{2}\right\} d x d t+ \\
& +\int_{\partial Q} e^{-\lambda t}\left\{K\left(w_{t t}^{2}+w_{t x}^{2}+w_{x x}^{2}\right)\right\} v_{0} d s+\int_{\partial Q} e^{-\lambda t}\left\{K w_{t t} w_{t x}+2 \alpha w_{t} w_{t x}+w_{x t} w_{t t}\right\} v_{1} d s=\sum_{i=1}^{3} J_{i}
\end{aligned}
$$

where $J_{1}$ is integral along the domain and $J_{i}, i=2,3$ are integral along the boundary. Considering the condition of Theorem 2, using Young's inequality, results in:

$$
J_{1}=\varepsilon\left\|\frac{\partial \Delta w}{\partial t}\right\|_{0}^{2}+m \int_{Q} \exp (-\lambda t)\left[w_{t t}^{2}+w_{t x}^{2}+w_{x x}^{2}\right] d x d t \leq \text { const }
$$

Based on boundary conditions in Eqs. (14),(15) and the condition of Theorem 2,

$$
J_{i}=0, i=1,2 .
$$

$c=$ const does not depend on $N$, hence from Eqs. (17)-(22) the second estimate for the approximate solution of Eqs. (6)-(8) follows. An estimate of Eq. (17) together with Eq. (21) allows to pass to limit at $N \rightarrow \infty$ and conclude that a subsequence $\left\{u_{\varepsilon}^{N_{k}}\right\}$ converges in view of the uniqueness (Theorem 1 ) in $L_{2}(Q)$ together with a first-and second-order to the desired regular solution $u_{\varepsilon}(x, t)$ of Eqs. (6)-(8) possessing the properties stated in Theorem 2 [6, 8, 10, 13, 14].

For $u_{\varepsilon}(x, t)$ by virtue of Eq. (21), the inequality holds true

$$
\varepsilon\left\|\frac{\partial}{\partial t} \Delta u_{\varepsilon}\right\|_{0}^{2}+\left\|u_{\varepsilon}\right\|_{2}^{2} \leq m\left[\|f\|_{0}^{2}+\left\|f_{t}\right\|_{0}^{2}\right]
$$

Theorem 2 is thus proven.

Now using the method of " $\varepsilon$-regularization" the solvability of Eqs. (1)-(3) is proven.

\section{Theorem 3:}

Let all conditions of Theorem 2 be fulfilled. Then the generalized solution of the problem from $W_{2}^{2}(Q)$ exists and is unique. 


\section{Proof:}

The uniqueness of the solution of problem in Eqs. (1)-(3) from $W_{2}^{2}(Q)$ is proven in Theorem 1. Now the existence of a generalized solution of Eqs. (1)-(3) from $W_{2}^{2}(Q)$ is proven. For this, consider Eq. (6) in domain $Q$ with boundary conditions in Eqs. (7), (8) at $\varepsilon>0$. Since all conditions of Theorem 2 are fulfilled, then there exists a unique regular solution of problem Eqs. (6)-(8) at $\varepsilon>0$ and for this solution estimates I), II) are true.

From here follows, under the known theorem of weak compactness, that from a set of functions $\left\{u_{\varepsilon}\right\}, \quad \varepsilon>0$ it is possible to take a poorly converging sub-sequence of functions in $V$ such that $\left\{u_{\varepsilon_{i}}\right\} \rightarrow u \quad$ at $\quad \varepsilon_{i} \rightarrow 0$. It will be shown that limiting function $u(x, t)$ satisfies the equation $L u=f(1)$. In reality, as the sequence $\left\{u_{\varepsilon_{i}}\right\}$ converges poorly in $W_{2}^{2}(Q)$ and the sequence $\left\{\frac{\partial^{3} u_{\varepsilon_{i}}}{\partial t^{3}}\right\},(\varepsilon>0)$ is in regular intervals limited in $L_{2}(Q), \quad$ and the operator $L-$ linear it is had $L u-f=L u-L u_{\varepsilon_{i}}+\varepsilon_{i} \frac{\partial \Delta u_{\varepsilon_{i}}}{\partial t}=L\left(u-u_{\varepsilon_{i}}\right)+\varepsilon_{i} \frac{\partial \Delta u_{\varepsilon_{i}}}{\partial t}$

From here, the following inequality will be performed

$$
\|L u-f\|_{0}=\left\|L\left(u-u_{\varepsilon_{i}}\right)\right\|_{0}+\varepsilon_{i}\left\|\frac{\partial \Delta u_{\varepsilon_{i}}}{\partial t}\right\|_{0}, m\left\|u-u_{\varepsilon_{i}}\right\|_{2}+\varepsilon_{i}\left\|\frac{\partial \Delta u_{\varepsilon_{i}}}{\partial t}\right\|_{0}
$$

From (25) approaching a limit at $\varepsilon_{i} \rightarrow 0$ we get the unique solution of Eqs. (1)-(3). Thus, theorem 3 is proven.

\section{SMOOTHNESS OF THE SOLUTION}

Now, the more general case of $l . .3$ must be proven. Further, the coefficients of Eq. (1) are assumed to be infinitely differentiable in a closed domain $\bar{Q}$.

\section{Theorem 4:}

Let the conditions of Theorem 3 be fulfilled and let $2\left(\alpha+m K_{t}\right)-\left|K_{t}\right|+\lambda|K| \geq \delta>0$. Then, for any function $f(x, t)$ such that $f \in W_{2}^{m}(Q), D_{t}^{m+1} f \in L_{2}(Q),\left.\quad \gamma D_{t}^{m} f\right|_{t=0}=\left.D_{t}^{m} f\right|_{t=T}$, there exists a unique generalized solution to problem (1)-(3) from the space $W_{2}^{m+2}(Q)$, where $m=0,1,2,3, \ldots$

\section{Proof:}

From the smoothness of the solution to problem in Eqs. (9)-(13), the following conditions for the approximate solution of Eqs. (6)-(8) follow:

$$
\begin{gathered}
w=u_{\varepsilon}^{N} \in C^{\infty}(Q) ;\left.\quad \gamma D_{t}^{q} w\right|_{t=0}=\left.D_{t}^{q} w\right|_{t=T} ; q=0,1,2,3,4, \ldots \\
w(0, t)=w(1, t)=0 .
\end{gathered}
$$


Considering the conditions of Theorem 2 at $\varepsilon>0$, nonlocal conditions at $t=0, t=T$ and from the equality

$$
\left.\left(e^{-\frac{\lambda t}{2}} L_{\varepsilon} u_{\varepsilon}\right)\right|_{t=0} ^{t=T}=\left.\left(-\varepsilon e^{\frac{-\lambda t}{2}} \frac{\partial}{\partial t} \Delta u_{\varepsilon}+e^{\frac{-\lambda t}{2}} L u_{\varepsilon}\right)\right|_{t=0} ^{t=T}=\left.\left(e^{\frac{-\lambda t}{2}} f(x, t)\right)\right|_{t=0} ^{t=T}
$$

then

$$
\left\|\gamma \cdot u_{\varepsilon t t t}(x, 0)-u_{\varepsilon t t t}(x, T)\right\|_{0} \leq \text { const }
$$

Hence, it follows that function $v_{\varepsilon}(x, t)=u_{\varepsilon t}(x, t)$ belongs to $V$ and satisfies the following equation

$$
P_{\varepsilon} v_{\varepsilon}=L_{\varepsilon} v_{\varepsilon}+K_{t} v_{\varepsilon_{t}}=f_{t}-\alpha_{t} u_{\varepsilon t}-c_{t} u_{\varepsilon}=F_{\varepsilon}
$$

From Theorem 2, it follows that the set of functions $\left\{F_{\varepsilon}\right\}$ is uniformly bounded in the space $L_{2}(Q)$, i.e.

$$
\left\|F_{\varepsilon}\right\|_{0} \leq m\left[\|f\|_{0}^{2}+\left\|f_{t}\right\|_{0}^{2}\right]
$$

Further, from the conditions of Theorem 3, one can easily see that operators $P_{\varepsilon},(\varepsilon>0)$ satisfy the conditions of Theorem 4. From here, based on estimates of I) and II) for a function $\left\{v_{\varepsilon}\right\}$, the following analogical estimates are obtained

$$
\begin{gathered}
\varepsilon\left(\left\|\frac{\partial^{2}}{\partial t^{2}} v_{\varepsilon}\right\|_{0}^{2}+\left\|\frac{\partial^{2}}{\partial t \partial x} v_{\varepsilon}\right\|_{0}^{2}\right)+\left\|v_{\varepsilon}\right\|_{1}^{2} \leq m\left(\|f\|_{0}^{2}+\left\|f_{t}\right\|_{0}^{2}\right) \\
\varepsilon\left\|\frac{\partial}{\partial t} \Delta v_{\varepsilon}\right\|_{0}^{2}+\left\|v_{\varepsilon}\right\|_{2}^{2} \leq m\left[\|f\|_{1}^{2}+\left\|f_{t t}\right\|_{0}^{2}\right]
\end{gathered}
$$

Function $\left\{u_{\varepsilon}\right\}$ satisfies a parabolic equation with conditions in Eqs. (2),(3)

$$
u_{\varepsilon}=u_{\varepsilon t}-u_{\varepsilon x x}=f+\varepsilon \frac{\partial}{\partial t} \Delta u_{\varepsilon}-K u_{\varepsilon t t}-(\alpha-1) u_{\varepsilon t}-c u_{\varepsilon}=\Phi_{\varepsilon},
$$

here $\Phi_{\varepsilon} \in L_{2}(Q)$. The set of functions $\left\{\Phi_{\varepsilon}\right\}$ is uniformly bounded in $W_{2}^{1}(Q)$ i.e.

$$
\left\|\Phi_{\varepsilon}\right\|_{1}^{2} \leq m\left[\|f\|_{1}^{2}+\left\|f_{t t}\right\|_{0}^{2}\right] \leq m\|f\|_{2}^{2}
$$

Based on priory estimates for parabolic equations $[4,6]$ and inequality in Eq. (30), then

$$
\left\|u_{\varepsilon}\right\|_{3}^{2} \leq m\|f\|_{2}^{2} \text {. }
$$

Similarly, one can prove that

$$
\left\|u_{\varepsilon}\right\|_{m+2}^{2} \leq m\|f\|_{m}^{2}
$$

where $m=2,3, \ldots$ 


\section{CONCLUSION}

Solvability of the problem considered can be formulated in the terms of smoothness of $K(x, t)$ in the case where, $K(x, 0) \leq 0 \leq K(x, T)$. In this the second kind of mixed type equation studied, since there is no restriction inside the domain. Under the assumption that the coefficients of the equation are smooth enough and $\alpha(x, t), c(x, t)$ are periodic with respect to variable $t$.

\section{REFERENCES}

[1] Alimov NA. (1983) On a nonlocal boundary value problem for a non-classical equation. The theory and methods for solving ill-posed problems and their applications. Novosibirsk, 237-239.

[2] Berezinsky YM. (1965) Expansion in eigenfunctions of selfadjoint operators. Kyev.

[3] Berdyshev AS. (1993) Nonlocal boundary value problems for equations of mixed type in a deviation from the characteristics. Differensial.Uravn., 29(12):2118-2125.

[4] Bitsadze AV, Samarskii AA. (1969) On some simple generalizations of linear elliptic boundary value problems. DAN SSSR, 185(4):793-740.

[5] Vragov VN. (1983) Boundary problems for non-classical equations of mathematical physics. Novosibirsk, 216pp.

[6] Glazatov SN. (1985) Nonlocal boundary problems for mixed type equations in a rectangle. Siberian Math. J., 26(6):162-164.

[7] Djamalov SZ. (1989) On correctness of nonlocal boundary problems for many-dimensional mixed type equation. Application of method of functional analysis to the non-classical equations of mathematical physics. Novosibirsk, 63-70.

[8] Yogorov IE. (1995) On smoothness of a solution to a nonlocal boundary value problem for an operator-differential equation with variable time direction. Mat. Zametki YaGU, 2(1):98104.

[9] Il'in VA, Moiseev YI. (2000) On the uniqueness of solution of mixed problem for wave equation with nonlocal boundary conditions. Differens. Uravn., Ç6(5):656-661.

[10] Karatkoprakliev GD. (1987) Nonlocal boundary problems for mixed type equations. Differens. Uravn., 23(1)78-84.

[11] Karatopraklieva MG. (1991) A nonlocal boundary-value problem for an equation of mixed type. Differens. Uravn., 27(1):68-79.

[12] Kalmenov TS. (1990) On the Dirichlet problem and nonlocal boundary problems for wave equation. Differens. Uravn., 26(1):60-65.

[13] Kozhanov AI. (1990) Boundary problems for equations of mathematical physics of odd order. Novosibirsk, $132 \mathrm{pp}$.

[14] Kuzmin AG. (1990) Non-classical mixed type equations and their applications to the gas dynamics. Leningrad 271pp.

[15] Ladyjenskaya OA. (1973) Boundary problems of mathematical physics. Moscow $470 \mathrm{pp}$.

[16] Moiseev YI. (2001) On a solvability of a nonlocal boundary problem. Differens. Uravn., 37(11):1565-1567.

[17] Terekhov AN. (1985) Nonlocal boundary problems for equations of variable type. Nonclassical equations of mathematical physics. Novosibirsk, 148-158.

[18] Nakhushev AM. (1982) On an approximate method for solving boundary value problems for differential equations and its application to the dynamics of soil moisture and groundwater. Differens. Uravn., 18(1):72-84.

[19] Beilin SA. (2006) On a mixed nonlocal problem for a wave equations. Electronic J. Diff. Equations, 103:1-10.

[20] Berdyshev AS, Karimov ET. (2006) Some-non-local problems for the parabolic-hyperbolic type equation with non-characteristic line of changing type. Central European J. Math. (CEJM), 4(2):183-193. 
[21] Rassias JM, Karimov ET. (2010) Boundary-value problems with initial non-local condition for parabolic equations with parameter. Eur. J. Pure Appl. Math. (EJPAM), 3(6):948-957. 